festerait en été pour compenser la diminution de la densité et de la friction intérieure de l'eau. Celte friction diminuant d’à peu près moitié quand la température s'élève dé $0^{\circ}$ à $95^{\circ}$ (C., la tendance à l'enfoncement. croît proportionnellement (C. Westromero-Lunu, sgoo el rg96; Os'Jwaton, tgot). D'autres biologistes souliennont que lo role des appendices de suspension est tout différent; ils y voient des appareils d'équilibration assurant la díreclion des mouvements des Cladocèros, régularisant leur nage, de façon à les maintenir, à un niveau déterminé, dans les couches d'eau qui sont supposées leur offrir les ressources alimentaires les plus abondantes ( $R$. WorJerisck, rgг3).

(A suivre).

\title{
LES GRILLES ÉLECTRIQUES \\ AUX ÉTATS-UNIS \\ PROGRÈS RÉALISÉS EN 1930
}

\author{
par M. GALLOIS \\ Conservateur des Eaux et Forêts en retraite \\ et M. DE DROUIN DE BOUVILLE \\ Inspecteur principal des Eaux et Forêts en retraite
}

En r 9 r3, les autorités de Lafayette, dans l'état d'Indiana, curent leur attention attirée par l'anormale abondance des poissons d'eau douce apportés au marché de la ville. Une enquête fut ouvertc et on découvrit, près d'un pont de la rivière Wasbash, affluent de l'Ohio, deux pêcheurs dont le manège ne laissait pas d'être singulier. Le premier, sur la rive, tenait en main une ligne montée sur une très longue gaule ; le second, slationnant un peu en aval sur une barque, recueillait au moyen d'une épuiselle des quantités massives de poissons pâmés.

En y regardant de près on s'aperçut que la ligne était un fil métallique très fin, soigneusement isolé, qui étail mis par intervalles en contact avec unc canalisalion électrique aéricnne : celle d'un tramway. Ainsi se trouvait dérivé un courant do 50 volls qui huait ou élourdissait les poissons dans un certain rayon (I).

Ce fait divers semble bien etre la relation de la première application de l'électricité à l'halieulique, donl dérivo vraisemblablement l'irlóc qu'un

(1) La pêche électrique; - Bullelin suisse de pêche el Piscicullure, Neuchâtel ; $N^{\circ}, 1$, Janvier 1914, p. 18 .

Cet arlicle - qui nous a été signalé par M. ne Lagirabrivide, Conservaleur des Laux el lorels - se réfere, sins aulre próteision, ì l'electrical World. 
recours à cette même force permeltrait d'interdire aux poissons l'accès des dérivations où ils se fourvoient.

En tout cas, eenx qui curent les premiers colle icléc, dépourvus de conmajssances suffisantes, éprowvèrent une sépe de mécomples. 11 fallut. abendre rgab pour voir commenem les recherehes méthodiques qui conduisirent anx résultals positil's.

Il a déjà été renclu compte avec détail des investigations ou réalisations antérieures a 1929 (1). Une publication vient de sortic des presses, sous la signature de M. Elmar Hagarss (2) exposant les progrès réalisés en ra3o par la technique des grilles électriques. Nous lui empruntorons tous les renseignoments nécessaires pour metlre au courant ceux qui, en France, s'intéressent à ces appareils nouveaux. Ils devraient être nombreux, càr pêcheurs et usiniers souhaitent également qu'on arrive à retenir le poisson en amont des prises d'eau sans arreter les feuilles ou herbes entrainées, ni créer une perte de charge. Comme l'a déclaré M. Fiscri, Sccrélaire général de l'Union électrique, à une séance récente de Commission au Ministère de l' $\Lambda$ griculturc où il fut parlé de l'invention américaine : - "Celte solution scrait très avantageuse pour l'industrie hydro-électrique. "

Rappelons, pour la compréhension de ce qui suit, que le Service des Pêches à Washington s'est assigné trois objectifs :

I $^{\circ}$ Empêcher les jeunes Salmonides (tacons) qui dévalent vers la mer de s'engager dans les artères maîtresses des réseaux d'irrigation ou les canavx d'amenée des usines hydrauliques ;

$2^{\circ}$ Interdire aux adultes de ces migraleurs potamotoques, lors de leur montée nuptiale, l'entrée des canaux de fuite de ces mêmes usines ;

$3^{\circ}$ Imposer aux poissons se déplaçant dans un bief un itinéraire sóterminé ; spécialement les diriger vers un piège ou le compartiment inférienr d'une échelle.

Rappelons aussi que la grille élcctrique est constituée : - soit par une frange d'électrodes tendue en surface avec tuyau ou chaine gisant sur fond (système Burkey) ; - soit par deux rancées parallèles d'électrodes, scmblables, sans ground elemenl (système Mac Millan dit encore "isolé "). Dans tous les cas, on dirige sur ces dispositifs un courant alternalif à la tension de $60-80$ volts.

Le problème posé par les prises d'eau agricoles et industrielles a été rapidement résolu.

Pour les premières, on a enregistré à nouveau, en sq.3o, l'efficacité des screcns placés en tête des canaux d'irrigalion de Sunnyside, Ticton et Wapato.

(1) Nouveaux dispositifs pour interdire aux Poissons laecis des dérivations hydrau-

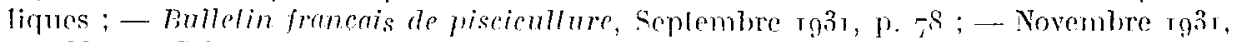
1. 133 ; - Dícembre т $93 \mathrm{r}$, ก. 169 .

(9) Progress in bidogicul inmiries, appendix III to report of Commissioner of Fisheries for the fiscal yerr 1930; - Covermmenl prinling Office, Washinglon, тg3т. 
Toutefois, sur ce dernier, une modification s'est imposée. La grille initiale étant trop rapprochée des vannes, une forte succion s'exerçait sur les Poissons qui s'en approchaient. Aussi se résolut-on à la déplacer vers l'amont. On profita de l'occasion pour l'améliorer en supprimant l'ćlément de fond. Celte modification a, comme ailleurs, été avantageuse, mais le bon fonctionnement conséculif est surtout imputable au fait que, maintenant, les tacons n'ayant plus à lutter contre un courant violent, ont toutes facilités pour gagner le canal de raccordement.

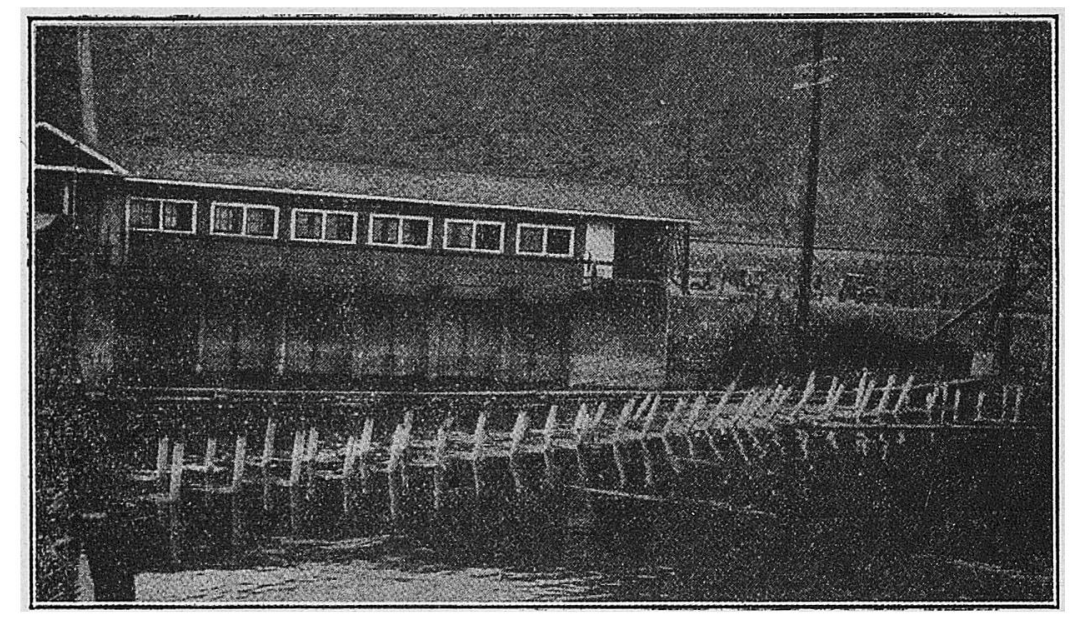

FIG. 52. - Grille électrique du Canal Wapatox Seconde installation, comportant deux rangées d'èlectrodes (1)

Sur le canal usinier de Gold Ray, la grille à double rangée d'électrodes, dont l'installalion avait été achevée à la fin de Septembre 1929 , fut remise en service au printemps suivant, mais, en raison de travaux, le fonctionnement se trouva interrompu au moment où le dévalage de tacons battait son plein. L'efficacité de l'appareil, si vraisemblable soit-elle, ne peut encore être affirmée. Mais une installation similaire a été achevée en I 930 , c'est celle de la Compagnie du Pacific Power and Light sur le canal Wapatox, dérivation de la rivière Nachez, près de Yakima (Etat de Washington). Le débit prélevé est d'environ 17 mètres cubes seconde. Il y a un élément de fond, constitué par un tuyau métallique.reposant sur un fond bétonné. La grille, placée peu au-dessus des vannes d'admission, à proximilé d'une décharge qui offre, pendant la belle saison, un excellent exutoire aux poissons, a donné pleine satisfaction. Le contrôle exercé permet d'avancer que, durant la période la plus aclive de la migration, $90 \%$ des tacons mal engagés ont élé arrêtés et remis en droit chemin.

En tant qu'obstacle au dévalage, la grille électrique a donc fait ses preuves aux Etats-Unis, mais on se propose maintenant de l'améliorer et simplifier.

(3) A comparer avec la figure 24. - Bulletin, Décembre т $3 \mathrm{~s}, \mathrm{p}$. гтr: 
En 1930, les plus importantes de ces recherches de perfectionnement onl élé dirigées par le Prolesseur Mac Mulaai el M. Wagner ; elles curent licu dans l'Old Indian Cianal, dérivalion de la Yakima, en ulilisant des alevins de Saumons chinook (Oncorhynchus tschawytscha Walbaum) longs de ro centimètres. Elles établirent :

I La supériorité de la grille isolée (insulated), constituée par une double frange d'électrodes, sur celle n'en comportant qu une rangée avec élément de fond ;

$2^{\prime}$ L'avantage des tuyaux de fort diamètre sur les chaînettes pour la conficction de ces électrodes ;

$3^{\circ}$ L'efficacité du courant ordinaire des réseaux de force et lumière, allernalif à 60 périodes, sans rccours aux transformations (interruption ct renverscment) d'abord préconisées.

D'autres investigations, poursuivies dans l'Oregon, firent comnaître les moilleurs espacements cntre électrodes et la tension la plus recom. mandable.

On utilisa l'appareillage ayant servi aux premiers essais à Gold Ray, après l'avoir transporté à l'élablissement de pisciculture de Fort Klamath, et on expérimenta sur de jeunes Saumons de fontaine (Salvelinus fontinalis Mitchill), ayant 5 cenlimètres de longueur, très vigoureux.

Les résultats les mcilleurs furent enregistrés avec une tcnsion de 55 volts, les électrodes élant distantes de $I^{111} 22$ et leurs rangées espacées de ${ }^{\mathrm{m}} 83$.

G'est en se basant sur ces données qu'ont élé établis les projets de grilles électriques pour prises d'cau à exéculer cu I 93 .

Comme installation au débouché des canaux de fuite, on n'a encore réalisé que celle de Gold Ray sur le Rogue river. Terminée seulement en Septembre r 929 , elle fonctionna fort peu au printemps 1930 , l'exploitation hydro-électrique ayant été suspendue daus les premiers jours de Mai pour cause de travaux. Et, quand la marche fut reprise, on s'aperçut que beau* coup de Saumons Chinook et Tête d'acier (Salmo Gairdneri Richardson) franchissaient l'obstacle, landis que certains élaient foudroyés au moment où ils passaicnt au-dessus du ground element. Ici encore se manifesta l'infériorité du système Burkey auquel on renonça, pour lui substitucr celui de Mac Millan déjà adopté l'année précédente pour le canal d'amenée. On tendit deux câbles parallèles séparés par un intervalle de ${ }^{m} 83$ et on y suspendit des tuyaux de fer galyanisé de ró centimètres de diamètre distants de $x^{m} 22$. Pour assurer leur immersion, en position approximativement verticale, ils sont lestés à la partie inférieurc d'une coupellc hémisphérique en tôle remplie de ciment. Le courant, alternatif de fréquence 6o, est modifié par le transformateur précédemment utilisé.

Avec cel aménagement, le succès fut complet. Les conditions d'observation étant favorables, on pul suivie les allures de centaines de reproducteur's en montée nuptiale; au contact du champ électrique ils rebrous. saient chemin et regagnaient la Rogue river. 
Lette réussile, pour brillante qu'elle soil, élant isoléc, appelle conlirmation.

Les essais d'cmploi de grilles électriques pour imposer aux poissons ccrtains trajels, entrepris en 1928 à Gold Ray, avaient abouti à un échec. ils furent repris en $\mathrm{I} 930$, le Bureau of Fisheries $\mathrm{y}$ attachant avec raison une grande importance.

Une première expérience cut lieu en Alaska, où on contrôle la remonte dans certains tributaires de la mer de Behring en coupant le passage aux migrateurs par de fortes grilles en bois dans lesquelles sout ménagées des

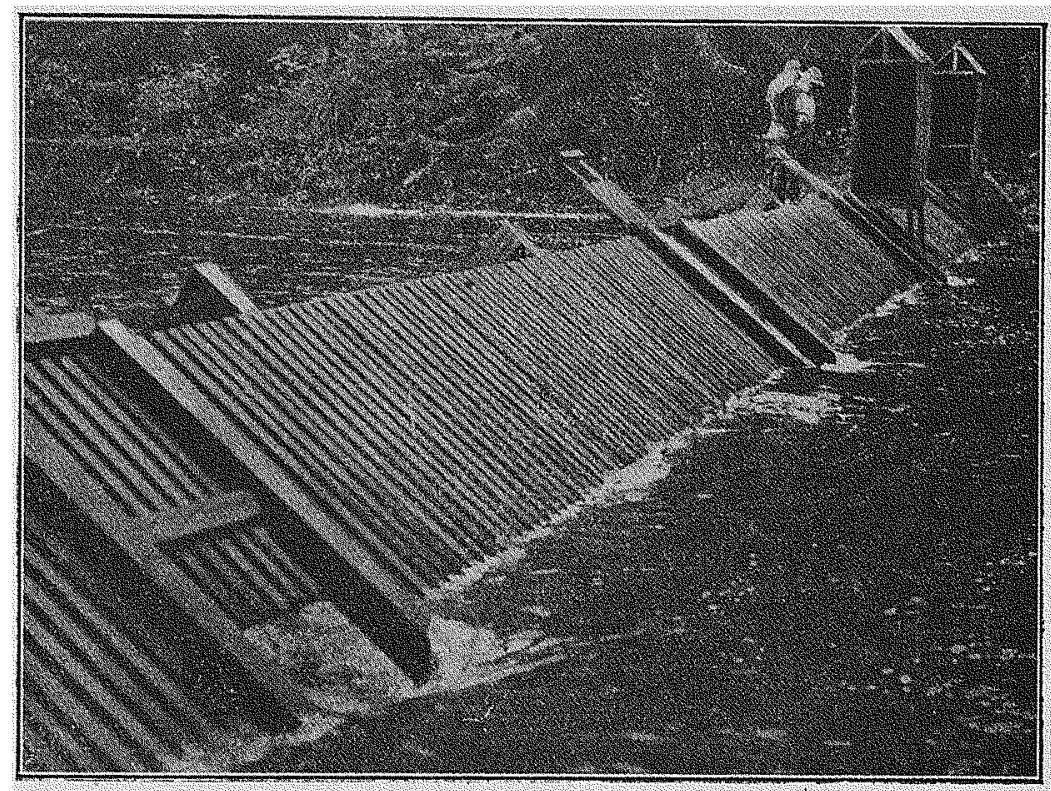

Liti. 53. .. Barrage pour captage des saumons de remonte Whole Passage (Alaska, 1929)

ouvertures; les poissons étanl contraints de les franchir, il esi possible d'en faire le recensement ( $\mathbf{r}$ ).

Mais, sur la rivière Kvichak, le dispositif habituel s'était engorgé cn I929 à raison de l'abondance des herbes ct algues entrâinées par lo courant. Pour parer à cet inconvénient, on imagina de réduire lc barrage de contrôle (counling weir) à sa partie centrale ct de dirigger les diverses montées (2) vers cel obstacle partiel au moyen d'unc vaste grille électrique. D'un dóveloppement de 230 mètres, elle offrait, en plan, la forme d'un V, dont la pointe, diriçée vers l'amont et ouverte sur une largeur de $\mathrm{I}_{2}{ }^{\mathrm{m}}: 20$, béait à 61 mètres au-dessous de l'estacade.

L'installation comprenait un câblc, soutenu par des picux, aựel pen-

(1) Voir Wann T. Thmsn: - Alasla fishory and far-seal indushries in 1929. - Covermment printing Oflice, Washinglon, rg3i, 1. 263.

(2) Chum Salmon : Oncorhynchus kela Walbnum ; - Pink Salmon : O. gorbuscha Walbauns ; - Silver Salmon : O. lisistch Walbaum. 
daient des chainclles, el un luyau reposanbsur le fond du lit. Ene dynamo de 9 kilowalls, actionnéc par: un moleur à essence de r5 CV., élait installée sur une rive, fournissant un courant alternatif de fréquence 60 à la tension de 6o-8o volts.

Dans son ensemble, la remonle fut arrêtée ; si quelques Saumons parvinrent à franchir l'ouvrage, plusicurs centaines de mille firent tête à (queue au contact du champ électríque, puis stationnèrent en aval. Par conséquent, le but qu'on s'était proposé ne fut pas atteint.

Pourquoi le poisson ne fut-il pas dirigé sur la pointe du V ? On n'a pu le délerminer ; il a élé obscrvé seulement que les passages destinés au comptage ne dommaient issue qu'à un faible courant d'eau, sans eflet allractif.

Aux Etals-Unis même, lc Bareau of Fisheries, cn collaboration avec la Northwestern Electric Lighl and Power Association, lit procéder durant l'élé de 1930, à des expériences qui eurent lieu à l'établissement de pisci. culture de la Green River. L'emplacement était bien choisi, car les montées de migrateurs y ont beaucoup d'importance et on y disposait d'une pêcherie, normalement ulilisée pour la capture des géniteurs dont on ulilise les produits sexucls pour la fécondation arlificielle. C'est immédiatement en aval qu'on disposa la grille électrique. Elle comprenait deux rangées d'électrodes qui élaient exactement aux écartements préconisés à la suite des recherches eflectuées à Fort Klamalh.

Pour micux apprécier s’il élait possible de guicler le poisson, on aménagea sur la rive est du cours d'cau, à I'cxlrémité de l'appareil, un réservoir en bois, à l’imitation du compartiment inférieur d'unc échclle, dans lequel on fit passer un fort courant d'eau.

Enfin, comme à ís mètres en aval de l'installalion, sur la même rive. débouche un petit ruisseau où remontent toujours quclques Saumons, on avait toute raison de penser que la grille électrique rabattrait vers cet affluent la majorité des migrateurs.

Cet espoir fut déçu ; toul au plus apcrcut-on de temps à autre une vingtaine de sujets s'ébattant dans le réservoir; quelques-uns aussi s'engagèrent dans le ruisscau, mais pas plus qu'antérieurement. Bref, comme en Alaska, on n'aboutit guère cu'ù arrèter la remonte.

Il est à noter que, pour ne pas porler préjudice à la migration descendante des lacons, il fallut réduire le voltage de 80 à $60-65$ volls.

Par conséquent, encore que M. Elmer Higgrss impute surtout Jes insuccès aux circonstances locales el fasse montro d'un cortain optimisme, il apparaîl que la crrille élcelrique est un obstacle qui ne se laisso pas tourner. Vraiscmblablement le champ déborde les électrodes extrimes ct les passages ou couloirs qu’on cnlend lajsser libres à la circulalion sont, en réalilé, interceptés par un réseau invisible de courant.

Il se peul qu'on arrive à surmonter celte difficullé, mais, pour le moment, elle scmble séricuse. 
La conclusion de l'anilyse qui précède est, qu’en peu dannées, le Service des pèches des Etats-tnis a alteinl deux sur trois des buls qu'î s'était assignés. On sait, maintenant, par un emploi bien réglé do l'énergic électrique,barrer efficacement aux migrateurs l'entrée ou la sortie de dérivations quelconque. C'est là un résultat fort important ct il est à présumer que les installations dont la techuique vient d'être mise au poim iront se multipliant. Souhailons gu'on ne tarde pas, en lirance, à recour'r à ce procédé pour maintenir el intensificr les courants de migration dans les bassins fluviaux où ils existent encore.

En terminant, signalons que quclques lignes du ripport de M. Eimen lliggins sont consacrécs aux grilles tournantes qui, sur les canaux de faible largeur, doivent c̀tre préférées aux appareils électriques. Elles confirment la salisfaction complète donnée par le modèle perf́cetionné de l'Oregon Game Commission, tel colui installé au commence. ment de rgiso sur le canal Ahtanum (1). L'unique donnée nouvelle est qu'il a été reconnu avantageux d'alimenter le canal de raccordement (by pass) par-un orifice noyé plutôt que par déversoir ; on lacilite ainsi au poisson l'accès de ce canal.

\title{
LES ASSOCIATIONS DE PEACHE
}

\author{
Par M. DE VAISSIERE \\ Garde général des Laux et Forèts, ì Rambouillet.
}

(Suite) ${ }^{(2)}$

b) Leur constitution. - Les associations de pèche, telles qu'elles sont conslituées à l'heure actuclle comprennent :

I $^{\circ}$ Des propriétaires, qui sont détenteurs du droit de pêcho et qui veulent en tirer profit ;

$3^{\circ}$ Des cultivateurs of fermicrs qui exploitent les ferres et qui jouissent très souvent du droit de pèche ;

$3^{\circ}$ Des pècheurs qui peuvent être des propriélaires, des rentiers, des ouvriers, des commerçants cl qui désirent s'adonner à leur sport favori.

Ordinairemenl, le droit de pêche se louc par bail : l'association communale peut devenir détentrice du droil de pèche en passant des baux avec chaque propriétaire. Ce système est très onéreux et donne énormément de peine. - La loi du 29 Juin rgis spécifie, en effet, que chaque bail doit Atre établi sur papier timbré cn trois exemplaires et enregistré, d'où multiplicité de baux à établir dans les petites propriétés. - En outre, l'artide 19 de la loi du 3 r Juillet r 920 soumet la location du droit de pêche à

(1) Voir Bulletin, Seplenbre s $93 \mathbf{1}$, j. 77 .

(2) Voir Bulletin, Mai 193 ?, p. 338. 